\title{
Competencia digital: cualidad ineludible para el actual desempeño profesional del docente universitario
}

\author{
Martha Ramona Cantero Ríos \\ martharamonacanterorios@gmail.com \\ Universidad Nacional De Pilar - FHCE \\ Pilar - Paraguay
}

\section{RESUMEN}

Las competencias digitales en el docente universitario se han vuelto un factor de gran importancia dentro del proceso de enseñanza desde hace varios años, sin embargo, con la crisis sanitaria desatada en el año 2020 a causa de la Covid-19, ha llegado a ser imprescindible. El presente trabajo se basó en una investigación documental de tipo observacional, descriptivo a partir de un enfoque cualitativo, con el propósito de identificar en la literatura existente el estado actual y la evolución de las competencias digitales, así como las perspectivas futuras de la educación superior. Para ello, como criterios de inclusión se propuso analizar libros y artículos científicos publicados entre los años 2010 a 2020, escritos en los idiomas español, inglés y portugués, publicados en revistas y bibliotecas virtuales especializadas en ciencias de la educación; dentro del proceso de búsqueda se emplearon los siguientes descriptores: alfabetización digital, competencias digitales, TIC, docencia universitaria. Además, se utilizó como base de datos a: ERIC, WEB of Science, Google Académico. Se escogieron 26 materiales bibliográficos, y, a través de una lectura crítica y objetiva, se ha obtenido como resultado la identificación del estado actual de las competencias digitales en docentes, la evolución por la cual han atravesado dichos docentes en cuanto a las exigencias sobre la utilización de las TIC en el desarrollo de las clases y la demanda que exigirá la educación superior en el futuro en cuanto a la actualización constante sobre las competencias digitales como cualidad ineludible del desempeño docente.

Palabras clave: educación superior; competencias digitales; TIC; docencia universitaria. 


\title{
Digital competence: an inescapable quality for the current professional performance of university teachers
}

\begin{abstract}
Digital competencies in university teachers have become a factor of great importance in the teaching process for several years, however, with the health crisis unleashed in 2020 due to Covid-19, it has become essential. The present work was based on a documentary research of observational, descriptive type from a qualitative approach, with the purpose of identifying in the existing literature the current state and evolution of digital competences, as well as the future perspectives of higher education. For this purpose, as inclusion criteria, it was proposed to analyze books and scientific articles published between 2010 and 2020, written in Spanish, English and Portuguese, published in journals and virtual libraries specialized in educational sciences; within the search process, the following descriptors were used: digital literacy, digital competencies, ICT, university teaching. In addition, the following databases were used: ERIC, WEB of Science, Google Scholar. Twenty-six bibliographic materials were chosen and, through a critical and objective reading, the result has been the identification of the current state of digital competences in teachers, the evolution through which these teachers have gone through in terms of the demands on the use of ICT in the development of classes and the demand that higher education will require in the future in terms of constant updating on digital competences as an inescapable quality of teaching performance.
\end{abstract}

Keywords: higher education; digital competencies; ICT; university teaching.

Artículo recibido: 10 Agosto. 2021 Aceptado para publicación: 07. Setiembre. 2021 Correspondencia: martharamonacanterorios@gmail.com Conflictos de Interés: Ninguna que declarar 


\section{INTRODUCCIÓN}

La implementación de las tecnologías de la información y comunicaciones (TIC) ha cambiado por completo el formato para el desarrollo de las clases dentro de la universidad. Esto se ha intensificado debido a pandemia de la Covid-19 que ha afectado al mundo entero, teniendo que optar por la virtualidad exclusiva para el desarrollo de las clases en el año 2020. En abril del año 2020, la Organización de las Naciones Unidas para la Educación, la Ciencia y la Cultura (UNESCO), ha lanzado una serie de recomendaciones sobre el traslado de las clases presenciales a virtuales, otorgando autonomía a cada universidad de proveer a los alumnos las plataformas adecuadas para la continuidad de las clases en línea (UNESCO, 2020). Debido a la crisis sanitaria aproximadamente 7.000.000 de niños y jóvenes han sido afectados por cierres de las instituciones educativas en más de 107 países (Hernández, 2020). En el caso de Paraguay, se han determinado fases de cuarentena parcial o total. Lo cual incluía el cierre de comercios no imprescindibles, y la permanencia activa de supermercados, farmacias y centros de salud. Por este motivo, las escuelas, colegios y universidades han sido clausuradas, pasando a dar las clases de forma totalmente virtual. Posteriormente, ya en el año 2020 fueron habilitadas algunas carreras en la modalidad híbrida que comprendía a las clases semipresenciales según lo permitía el Ministerio de Educación. Estas trasformaciones en el proceso de enseñanza-aprendizaje prácticamente ha obligado a la incorporación de modelos educativos innovadores acordes a la necesidad presente.

Por ello, el presente trabajo pretende realizar una revisión bibliográfica sobre las competencias digitales en docentes universitarios. Teniendo como objetivo principal el de describir el estado actual a partir de la literatura existente acerca de las competencias digitales en docentes universitarios, describir la evolución de las competencias digitales en un período de 10 años desde el año 2010 al 2020, así como la de describir las perspectivas futuras sobre la Educación Superior.

\section{ESTRATEGIAS METODOLÓGICAS}

El presente trabajo buscó realizar una revisión bibliográfica, de tipo descriptiva a partir de un enfoque cualitativo, con el propósito de identificar y describir las competencias digitales en docentes universitarios a partir de la literatura existente. Así como también, narrar sobre el estado actual de las competencias digitales en dichos docentes, además de describir la evolución de dichas competencias desde el año 2010 al 2020, y, por último, identificar las perspectivas futuras sobre la educación superior. 
Para el desarrollo del estudio se establecieron como criterios de inclusión: libros y artículos científicos de investigación teórica publicados entre los años 2010 a 2020, escritas en los idiomas español, inglés y portugués, publicados en revistas y bibliotecas virtuales especializadas en ciencias de la educación. Para el proceso de búsqueda se implementaron los siguientes descriptores: alfabetización digital, competencias digitales, TIC, docencia universitaria en bases de datos como: Base de datos ERIC, WEB of Science, Google Académico.

Para el análisis de los documentos seleccionados se realizó una lectura crítica y objetiva que posibilitó la identificación de bibliografías claves y de relevancia para la construcción del presente trabajo de investigación.

\section{RESULTADOS Y DISCUSIÓN}

\subsection{Definición de competencias digitales}

El Ministerio de Educación de España, en su Órgano del Instituto Nacional de Tecnologías Educativas y Formación del Profesorado (INTEF), aporta su propia definición al respecto de la competencia digital docente, en donde menciona que puede definirse, cómo: "el uso creativo, crítico y seguro de las tecnologías de información y comunicación para alcanzar los objetivos relacionados con el trabajo, la empleabilidad, el aprendizaje, el tiempo libre, la inclusión y la participación en la sociedad” (INTEF, 2017, p.9).

Según Pavié (2011, p. 77), la competencia digital "es un grupo de elementos combinados (conocimientos, destrezas, habilidades y capacidades) que se movilizan e integran en virtud de una serie de atributos personales, en contextos de acción". Por otro lado, Gutiérrez (2014), hace mención de que existen dos aspectos que se hacen énfasis en cuanto a las competencias digitales y son; los que resaltan el factor tecnológico, y los que destacan el informacional o comunicativo.

Así también, Lázaro \& Gisbert (2015, p. 325), afirman que la competencia digital docente es "la capacidad del profesorado de poseer un nivel de competencia digital que le permita utilizar la tecnología con eficacia, de forma adecuada y adaptada a sus estudiantes y a los aprendizajes de que éstos deben conseguir".

Cabe resaltar que la utilización de las tecnologías digitales no se centra únicamente en su uso correcto, sino que los docentes encargados de impartir clases a través de estas perciban el nivel de importancia y efecto que conlleva, ya que "pasa por la necesidad de comprender el profundo impacto de las tecnologías en un mundo digital y promover la colaboración para 
integrarlas de modo efectivo. La competencia digital es mucho más que solamente utilizar tecnologías digitales (Durán Cuarteto, Prendes Espinosa, \& Gutiérrez Porlán, 2019).

\subsection{Estado actual de las competencias digitales en docentes universitarios}

El Instituto Nacional de Tecnologías Educativas y de Formación del Profesorado (INTEF) considera que se debe adaptar y mejorar la educación por medio de la tecnología para emplearlo ya que puede ser útil para la cooperación y colaboración entre profesionales, alcanzar una calidad educativa y por sobre todo fomentar la equidad en la educación (INTEF, 2017). Así mismo, destaca que las competencias digitales son un conjunto de "conocimientos, habilidades y actitudes necesarias hoy en día para ser funcional en un entorno digital" (INTEF, 2017).

En el caso del docente universitario debe apropiarse de dichos conocimientos y habilidades para poder lograr un desempeño profesional de calidad. Sin embargo, todo depende de la actitud que cada profesor presente para logar adaptarse a la era tecnológica educativa y así poder interactuar con los alumnos y con otros profesionales (INTEF, 2017).

A continuación, se presenta una referencia detallada sobre las competencias digitales que deben poseer los docentes del Siglo XXI según lo plantea el INTEF (2017, p.2).

Tabla 1: Marco Común de Competencia Digital Docente

\begin{tabular}{|c|c|c|}
\hline $\begin{array}{c}\text { Áreas } \\
\text { competenciales }\end{array}$ & Competencias & Niveles competenciales \\
\hline $\begin{array}{l}\text { Área 1: } \\
\text { Información y } \\
\text { alfabetización } \\
\text { informacional }\end{array}$ & $\begin{array}{l}\text { Competencia 1.1. Navegación, búsqueda y } \\
\text { filtrado de información, datos y contenidos } \\
\text { digitales. } \\
\text { Competencia 1.2. Evaluación de información, } \\
\text { datos y contenidos digitales. }\end{array}$ & \\
\hline $\begin{array}{l}\text { Área } 2 . \\
\text { Comunicación y } \\
\text { colaboración }\end{array}$ & $\begin{array}{l}\text { Competencia 2.1. Interacción mediante las } \\
\text { tecnologías digitales } \\
\text { Competencia 2.2. Compartir información y } \\
\text { contenidos digitales. } \\
\text { Competencia 2.3. Participación ciudadana en } \\
\text { línea. } \\
\text { Competencia 2.4. Colaboración mediante } \\
\text { canales digitales. } \\
\text { Competencia 2.5. Netiqueta. } \\
\text { Competencia 2.6. Gestión de la identidad } \\
\text { digital. }\end{array}$ & \\
\hline
\end{tabular}




\begin{tabular}{|c|c|c|}
\hline $\begin{array}{l}\text { Área 3. Creación } \\
\text { de contenidos } \\
\text { digitales }\end{array}$ & $\begin{array}{l}\text { Competencia 3.1. Desarrollo de contenidos } \\
\text { digitales. } \\
\text { Competencia 3.2. Integración y reelaboración } \\
\text { de contenidos digitales. } \\
\text { Competencia 3.4. Programación }\end{array}$ & $\begin{array}{l}6 \text { niveles competeniales } \\
\text { por cada una de las } 21 \\
\text { competencias que } \\
\text { conforman el Marco. }\end{array}$ \\
\hline Área 4. Seguridad & $\begin{array}{l}\text { Competencia 4.1. Protección de dispositivos } \\
\text { Competencia 4.2. protección de datos } \\
\text { personales e identidad digital } \\
\text { Competencia 4.3. Protección de la salud } \\
\text { Competencia 4.4 Protección del entorno. }\end{array}$ & \\
\hline $\begin{array}{l}\text { Área } 5 . \\
\text { Resolución de } \\
\text { problemas }\end{array}$ & $\begin{array}{l}\text { Competencia 5.1 Resolución de problemas } \\
\text { técnicos. } \\
\text { Competencia 5.2. Identificación de } \\
\text { necesidades y respuestas tecnológicas. } \\
\text { Competencia 5.3. Innovación y uso de la } \\
\text { tecnología digital de forma creativa } \\
\text { Competencia 5.4. Identificación de lagunas en } \\
\text { la competencia digital. }\end{array}$ & \\
\hline
\end{tabular}

Fuente: (INTEF, 2017, p.2)

Por otra parte, Area (2010) plantea que el siglo XXI requiere de ciudadanos capaces de emplear las herramientas necesarias para poder desenvolverse dentro del sistema informacional y tecnológico actual, y que no abarca solo el aspecto educativo sino también el político, económico y social. Así mismo, García et al (2010), sostienen que las Tecnologías de la Información y las Comunicaciones (TIC) ya dan forma al proceso de construcción del aprendizaje, debido a que proponen las estructuras sobre las cuales se deben cimentar los planeamientos educativos.

Por ello, la UNESCO (2014), establece que el alcance que han logrado las TIC exige al sistema educativo una constante actualización en cuanto a las prácticas y a los contenidos que se deben impartir en las salas de aula, para que éstas se adecuen al nuevo entorno tecnológico informativo. Debido a que "los dispositivos informáticos y la comunicación digital posibilitan nuevas formas de representar, procesar, transmitir y circular el conocimiento; allanan el intercambio entre las personas independientemente de su situación geográfica" (Assinnato et al, 2018, p.51).

Sin embargo, desde el momento en que la información pueda circular por los dispositivos informáticos, significa que el conocimiento puede no necesariamente provenir de forma única del profesor al estudiante de manera vertical, y esto, puede representar un nuevo desafío para el docente universitario. Como lo menciona Pimmer, Mateescu, \& Gröhbiel 
(2016), no sirve de nada utilizar el programa de power point en vez de la pizarra, ni realizar las evaluaciones por medio de celulares si al final las premisas educativas no sufren de cambios.

Así mismo, Carrera \& Coiduras (2012, p. 274), mencionan que el caso de las universidades españolas asociadas al Espacio Europeo de Educación Superior (EEES), implementan nuevas e innovadoras estrategias metodológicas para el desarrollo de clases en el entorno universitario, teniendo en cuenta lo que ya se ha mencionado más arriba, sobre que los docentes ya no son la única fuente de información y conocimiento para los alumnos. Para ello, emplean instrumentos de evaluación no solamente a los exámenes escritos, sino que tienen a la "evaluación de la competencia en acción”. Así como también, la "atención tutorial basada en un seguimiento más cercano del aprendizaje del alumno" (Carrera \& Coiduras, 2012, p. 274)

Una de las realidades que se presentan es la falta de conocimientos requeridos en cuanto a las competencias digitales que deben emplear los docentes universitarios, en algunos casos, dichos maestros solicitan la ayuda de algún estudiante como auxiliar quien se encarga de realizar los procedimientos tecnológicos a través de los dispositivos informáticos, sin embargo, este hecho pone en desventaja al docente ante el alumnado (Castellanos Adarme, Nieto Sánchez, \& Parra López, 2018).

\subsection{Evolución de las competencias digitales en el período de 2010-a 2020.}

Según Ehuletche et al (2018, p.10), "el avance del paradigma tecnológico va generando la necesidad de repensar las políticas educativas". Esto se debe a la multiplicación de diversas fuentes de información, a la circulación del conocimiento en forma acelerada y al desarrollo de las tecnologías de información y comunicación. Sin embargo, dicho contexto desafía innegablemente a los docentes a repensar en la metodología aplicada para la enseñanza, a innovar en cuanto a los entornos de aprendizaje desde perspectivas diferentes.

Del año 2010 al 2020 ha existido un enorme avance en cuanto a las competencias digitales debido a que se ha pasado del desarrollo de clases con el apoyo de las TIC para complementar la información, a la educación virtual total prácticamente en todo el mundo. Ya que en el año 2020 debido a la pandemia desatada por la Covid-19, se ha implementado la educación a distancia utilizando los computadores y celulares como medios para el desarrollo de las clases. 
La idea que se tenía sobre las clases en el entorno universitario partía de la figura del docente enseñando a un auditorio cuantioso, con ayuda de fotocopias y apuntes. Sin embargo, en la actualidad los alumnos pueden acceder a los apuntes de las materias dentro del aula virtual. Además, las pizarras y fotocopias han sido reemplazadas por presentaciones proyectadas, $o$ por la pizza digital. Debido a esto los docentes que siguen impartiendo clases deben estar al día con las actualizaciones correspondientes en cuanto al manejo de las herramientas digitales. (Martín, Pérez, \& Jornano de la Torres, 2014)

Según Arias et al (2014, p. 356-357), trabajar utilizando las TIC "revolucionando el espaciotiempo-medio dentro o fuera del aula" en los espacios académicos puede ser relevante y determinante para la forma de comunicación que se da entre alumnos y docentes. Los mismos autores consideran que "los ambientes virtuales formarán el enlace o la red social intra-escolar que fortalecerán esta comunicación y que favorecerán el aprendizaje”.

Por otro lado, se presenta también un aspecto importante que todo docente universitario debe desarrollar que es la parte de la investigación. Además de ser profesor en aula, debe realizar publicaciones científicas y participar de congresos, entre otros. Esto requiere ineludiblemente la adquisición y capacitación constante de las competencias digitales (Bustos-González, 2019).

De acuerdo con Chaparro (2020, p.30), en el año 2020 debido a la pandemia y a causa del confinamiento vivido en el mundo entero, se ha desatado otro tipo de exigencia en cuanto al uso de las tecnologías en la educación, "ya no se habla de este como un complemento a la educación presencial, y es que así se entendía hasta hace poco la educación virtual y los recursos tecnológicos". En la actualidad, los medios digitales pasaron a ser los protagonistas del desarrollo educativo.

Desde el punto de vista de Ruiz-Ramírez et al (2020), el proceso de innovación vivenciado en la educación virtual a raíz de la crisis sanitaria por el coronavirus debe ser tratado como un acontecimiento de relevancia educativa debido a que pueden ser tomados los entornos virtuales como factores estratégicos que ayudan al desarrollo de un nuevo acceso a la información y a la creación de nuevos conocimientos. Así también, los mismos autores sostienen que, para la continuidad de las clases en línea, la exigencia de las competencias virtuales no solamente se inclina hacia el sector docente, sino que también a los alumnos ya que deben ser capacitados para poder ser parte de este nuevo método de enseñanzaaprendizaje (Ruíz-Ramírez et al, 2020). 
Como lo hacen notar Silva, Ramos Silva \& Montanari, (2020), una realidad de la educación actual es que los estudiantes de zonas rurales de diversos países de América Latina, han tenido la dificultad de adaptarse al modelo de enseñanza virtual, debido a la falta de herramientas tecnológicas y de buena señal de internet. Asimismo, Quevedo Ramírez (2020), expone que la mudanza del paradigma educativo del entorno presencial al virtual ha evidenciado grandes desigualdades que existen en países latinoamericanos en cuanto a la educación.

\subsection{Perspectivas futuras de educación superior}

Como señala Barrón (2020), en el caso de México, el 60\% de los estudiantes no poseen computadoras y acceso a internet. En el caso de América Latina, se denota la inexistencia de proyectos educativos a largo plazo en donde se tenga en cuenta a todos los sectores sociales. Todos estos avances tecnológicos no solo han afectado a la educación en sí sino también al ambiente familiar y laboral. Para la misma autora, la educación en línea "es necesaria pero insuficiente, sino se cambian los paradigmas educativos, si no se hace un análisis profundo de los currículos, (...) de la enseñanza, del aprendizaje y de la evaluación, de la práctica docente (...)" (Barrón, 2020, p. 70).

La enseñanza en el contexto de la educación superior debe indefectiblemente adaptarse a los nuevos contextos educativos. Sin embargo, entendiendo que la educación virtual permite el crecimiento económico de las instituciones de la educación superior, por medio de la reducción de costos en cuanto a infraestructura, servicios de energía eléctrica, entre otros, deben aprovechar la innovación tecnológica para aplicar nuevas metodologías en el aprendizaje y para el desarrollo de la investigación. Y que los ambientes virtuales no solo sean vistos como un negocio, que es unos de los problemas que podría surgir en el ámbito académico (Abad et al, 2020). 
Abad, et al (2020), plantean un esquema sobre los modelos tradicionales y actualizados de los entornos de aprendizaje. Entendiendo que las competencias digitales y la correcta utilización de las TIC, permitirán que el aprendizaje no sea conductista sino más bien constructivista, cambiando por completo el enfoque de la metodología educativa tradicional. Así como se observa en la siguiente tabla 2.

Tabla 2: Entornos de aprendizaje. Modelo tradicional y Actualizado

\begin{tabular}{|c|c|c|}
\hline Medio/Entorno & Modelo Tradicional & Modelo Actualizado (Evolución) \\
\hline Teoría del aprendizaje & $\begin{array}{l}\text { Conductismo } \\
\text { Cognitivismo }\end{array}$ & $\begin{array}{l}\text { Constructivismo social } \\
\text { Colectivismo }\end{array}$ \\
\hline $\begin{array}{l}\text { Adquisición del } \\
\text { conocimiento }\end{array}$ & Planificado & Flexible \\
\hline Aprendizaje & Lineal & Múltiple \\
\hline Enseñanza & Memoristica & Social \\
\hline $\begin{array}{l}\text { Gestor } \\
\text { Rol del docente }\end{array}$ & $\begin{array}{c}\text { Docente } \\
\text { Transmisor del } \\
\text { conocimiento }\end{array}$ & $\begin{array}{l}\text { Estudiante } \\
\text { Guia del conocimiento }\end{array}$ \\
\hline Organización & Asignaturas & Casos prácticos \\
\hline Metodologia & $\begin{array}{l}\text { Competitiva } \\
\text { Individualismo }\end{array}$ & $\begin{array}{c}\text { En grupo } \\
\text { Colaborativo } \\
\text { Asociacionismo }\end{array}$ \\
\hline TIC & Aula virtual & $\begin{array}{l}\text { Sitios web } \\
\text { (comunidad de usuarios) }\end{array}$ \\
\hline
\end{tabular}

Fuente: (Abad, et al, 2020, p. 97).

Desde la posición de Villavicencio et al (2020, p. 859), "la innovación consiste en repensar la práctica docente empleando los medios tecnológicos para potenciar el aprendizaje del alumno, y para ello la pieza clave es el docente". El profesor es quien creará un entorno formativo a través de las TIC, así como la “creación de redes de conocimiento”. Sin embargo, lo primordial en todo lo que implica la educación virtual es la accesibilidad de las TIC, para que todos los estudiantes puedan acceder a dicha tecnología, y se pueda evitar la marginación y segregación entre los estudiantes y docentes.

\section{CONCLUSIONES}

De acuerdo con los objetivos planteados, la literatura analizada ha demostrado el gran avance tecnológico que existe desde el año 2010 al 2020. Dicho avance ha propiciado un modelo de 
enseñanza constructivista. Sin embargo, con la educación virtual se evidencia la segregación entre los docentes y estudiantes que pueden acceder a las TIC, que poseen las competencias digitales requeridas para el nuevo paradigma y los que no, específicamente debido a razones económicas y geográficas.

Se ha logrado identificar el estado actual de las competencias digitales en docentes de la Educación Superior y las perspectivas futuras, dejando claro que el nuevo paradigma educativo de la enseñanza virtual ha venido para quedarse, lo cual obliga tanto a docentes como a alumnos a capacitarse constantemente para estar a la vanguardia con los conocimientos que se requieren para la obtención de las nuevas competencias.

Así también, se puede destacar que, para los docentes que actualmente están impartiendo clases permanezcan en sus puestos de trabajo, deben ineludiblemente adoptar esta nueva metodología de enseñanza a través de las TIC, basado en las competencias digitales, ya que, de no ser así, podrían perder sus empleos. Debido a que dichas competencias se han vuelto una obligatoriedad para los docentes en el sector de la Educación Superior.

\section{BIBLIOGRAFÍA}

Abad, E., González, M., \& Belmonte, L. (2020). Aprendizaje significativo en el desarrollo de competencias digitales. Análisis de tendencias. International Journal of Educational Research and Innovation, 91-110.

Abad-Segura, E., González, M., Luque de la Rosa, A., \& Gallardo , J. (2020). Gestión de la economía digital en la educación superior: tendencias y perspectivas futuras. Revista Campus virtuales, 57-68.

Area Moreira, M. (2010). ¿Por qué formar en competencias informaciones y digitales en la educación superior? Revista de Universidad y Sociedad del Conocimiento, 1-5.

Arias, M., Torres, T., \& Yáñez, J. (2014). El desarrollo de competencias digitales en la educación superior. Historia y Comunicación Social, 355-366.

Assinnato, G., Mateus, J., \& Novomisky, S. (2018). Las TIC en la enseñanza universitaria de la Comunicación: usos, sentidos y representaciones en dos universidad de Argentina y Perú. Catalunya: Communication Papers Media Literacy \& Gender Studies.

Barrón, M. (2020). La educación en línea. Transiciones y disrupciones. . Educación y pandemia, 66-74. 
Bustos-González, A. (2019). 1Tránsito de universidad docente a universidad de investigación. ¿Un problema de información académica, de axonomías o de rankings universitarios? El profesional de la información, 1-13.

Castellanos Adarme, M., Nieto Sánchez, z., \& Parra López, H. (2018). Interpretación delas competencias digitales profesorales en el contexto universitario. Revista Logos Ciencia / Tecnología, 41-51.

Chaparro, B. (2020). Las nuevas prácticas digitales de docentes de cursos artísticos en la educación superior en Latinoamérica a raíz de la pandemia COVID-19: Aproximaciones y experiencias. revista internacional de pedagogía e innovación educativa, 29-40.

Durán Cuarteto, M., Prendes Espinosa, M., \& Gutiérrez Porlán, I. (2019). Certificación de la Competencia Digital Docente: propuesta para el profesorado universitario. Revista Iberoamericana de Educación a Distancia, 187-205.

Ehuletche, A., Lado, S., Atlante, M., \& Malbernat, L. (2018). Competencias para el uso de tecnologías educativas de docentes de nivel superior. Análisis longitudinal del período 2012-2017 en América Latina. Virtualidad, Educación y Ciencia, 9-21.

García, A., Arras, A., González, L., Hernández, A., Martín de Arriba, J., Prada San Segundo, M., . . . Torres, C. (2010). Competencias en TIC y rendimiento académico en la universidad: diferencias por género. Salamanca: AECID.

Gutiérrez, I. (2014). Perfil del profesor universitario español en torno a las competencias digitales en tecnologías de la información comunicación. Píxel-Bit. Revista de Medios y Educación, 51-65.

Hernández, J. (2020). Impacto de la Covid-19 sobre la salud mental de las personas. ,. Medicent Electrón, 578-594.

INTEF. (2017). Common Digital Competence Framework for Teachers. Obtenido de http://blog.educalab.es/intef/2017/01/26/common-digital-competence-frameworkfor-teachers/

INTEF. (2017). Marco Común de competencia digital docente. Octubre 2017. España: Instituto Nacional de Tecnologías Educativas y de Formación del Profesorado.

Lázaro, J., \& Gisbert, M. (2015). El desarrollo de la competencia digital docente a partir de una experiencia piloto de formación en alternancia en el Grado de Educación. Revista Educar, 321-348. 
Martín, A., Pérez, L., \& Jornano de la Torres, M. (2014). Las competencias digitales docentes en entornos universitarios basados en el Digcomp. Cultura digial y educación, 1-21.

Pavié, A. (2011). Formación Docente: hacia una definición del concepto de competencia profesional docente. Revista electrónica interuniversitaria de Formación del Profesorado, 67-80.

Pimmer, C., Mateescu, M., \& Gröhbiel, U. (2016). Mobile and ubiquitous learning in higher education settings. A systematic review of empirical studies. Computers in Human Behavior, 1-21.

Quevedo Ramírez, E. (2020). Educación a distancia. Política educativa y escenario tecnosociológico venezolano en tiempo de Covid-19. Revista Educare, 308-322.

Ruíz-Ramírez, J., Tamayo-Preval, D., \& Montiel-Cabello, H. (2020). Competencias Digitales De Los Docentes En La Modalidad De Clases En Línea: Estudio De Caso En El Contexto De Crisis Sanitaria. Linguagem e Tecnología, 1-16.

Silva, T., Ramos Silva, E., \& Montanari, R. (2020). Dificultades de la educación remota en las escuelas rurales del norte de Minas Gerais durante la pandemia de Covid-19. Research, Society and Development, 1-14.

UNESCO. (2014). Enfoques estratégicos sobre las TIC en Educación en América Latina y el Caribe. Santiago: Organización de las Naciones Unidas para la Educación, la Ciencia y la Cultura.

UNESCO. (2 de abril de 2020). El Coronavirus Covid-19 Y La Educación Superior: Impacto $Y$ Recomendaciones. Obtenido de https://www.iesalc.unesco.org/2020/04/02/elcoronavirus-covid-19-y-la-educacion-superior-impacto-y-recomendaciones/

Villavicencio, J., Hernández, G., \& Soto, M. (2020). El Proceso Educativo Durante La Pandemia Covid19 En La Universidad Autónoma De Sinaloa. Movilidad virtual de experiencias educativas, 852-856. 